5. Fazeli A, Dickinson SL, Hermiston ML, et al. Phenotype of mice lacking functional Deleted in colorectal cancer (Dcc) gene. Nature 1997; 386 796-804

6. Mehlen P, Rabizadeh S, Snipas SJ, et al. The DCC gene product induces apoptosis by a mechanism requiring receptor proteolysis. Nature 1998 ; $395: 801-4$.
7. Mehlen P, Bonod-Bidaud C, Bordeaux MC, et al. La notion de récepteurs à dépendance, docteur Jekyll et M. Hyde. Med Sci (Paris) 2001 ; 744-52.

8. Mehlen P, Fearon ER. Role of the dependence receptor DCC in colorectal cancer pathogenesis. J Clin Oncol $2004 ; 22: 3420-8$.

9. Castets M, Broutier L, Molin Y, et al. DCC constrains tumour progression via its dependence receptor activity. Nature 2011 ; 482 : 534-7.
10. Watson AJ, Pritchard DM. Apoptosis in intestinal epithelium: lessons from transgenic and knockout mice. Am J Physiol Gastrointest Liver Physiol 2000 ; $278:$ Gl-5

11. Krimpenfort P, Song JY, Proost $\mathrm{N}$, et al. Deleted in colorectal carcinoma suppresses metastasis in $\mathrm{p} 53$ deficient mammary tumours. Nature 2012 ; 482 : 538-41.

\title{
NOUVELle
}

\section{Srf : un acteur clé de l'hypertrophie du muscle squelettique}

Aline Guerci, Charlotte Lahoute, Sophie Hébrard, Laura Collard, Dominique Daegelen, Athanassia Sotiropoulos
Inserm U1016, Institut Cochin, 22, rue Méchain, 75014 Paris, France ; CNRS UMR8104, 75014 Paris, France ; Université Paris Descartes, 75006 Paris, France. athanassia.sotiropoulos@inserm.fr

\section{Plasticité du muscle squelettique} Les muscles squelettiques représentent $50 \%$ de la masse corporelle totale. Outre leur activité contractile, ils sont le siège d'activités métaboliques et constituent le réservoir majeur de protéines dans l'organisme. Le muscle squelettique adulte est un tissu particulièrement plastique, capable d'adapter sa taille aux signaux environnementaux et à diverses sollicitations comme les surcharges fonctionnelles, l'activité neuronale, les hormones et la disponibilité en nutriments. Il est de plus doté d'une capacité de régénération en cas de lésion. Chaque muscle squelettique est constitué de myofibres postmitotiques plurinucléées et d'une minorité de cellules souches mononucléées, les cellules satellites.

Les myofibres matures peuvent croître via l'addition de nouvelles protéines contractiles aux sarcomères préexistants. Ce mécanisme requiert l'augmentation de la synthèse protéique via la voie de signalisation PI3K/Akt/mTOR (target of rapamycin). L'accumulation et la perte de protéines contractiles dans les fibres musculaires sont associées respectivement à l'hypertrophie et à l'atrophie [1]. Les myofibres peuvent également croître via l'acquisition de nouveaux noyaux obtenue par la fusion avec des cellules satellites. $\varepsilon$ n effet, en réponse à différents stimulus, les cellules satellites peuvent être activées, ce qui induit leur prolifération, puis leur engagement dans la voie myogénique et leur fusion avec la fibre adulte en croissance. Les fonctions des cellules satellites sont contrôlées par des facteurs extrinsèques tels que des facteurs de croissance et des cytokines [2]. En particulier, les myokines sécrétées par les myofibres telles que I'IL(interleukine)-6 et I'IL-4 modulent respectivement la prolifération et la fusion des progéniteurs musculaires $[3,4]$.

\section{Srf (serum response factor)} et fonction des cellules satellites dans la réponse hypertrophique L'homéostasie musculaire est fortement perturbée au cours du vieillissement (sarcopénie) et dans de nombreuses situations pathologiques (immobilisation, cachexie). Il est donc essentiel de mieux comprendre les mécanismes cellulaires et moléculaires responsables de la croissance musculaire. Dans ce contexte, nous nous sommes intéressés au facteur de transcription Srf (serum response factor) qui est fortement exprimé dans les muscles squelettiques et contrôle notamment l'expression des gènes codant pour des protéines sarcomériques (actine squelettique, myosine).

L'utilisation de souris génétiquement modifiées, chez lesquelles il est possible d'invalider le gène Srf sélectivement dans le muscle squelettique, a d'abord permis de révéler le rôle crucial de ce facteur dans la croissance musculaire postnatale [5]. Mais chez l'adulte, quel pourrait être le rôle de Srf dans la plasticité musculaire et notamment dans le contrôle de la masse musculaire? D'une manière intéressante, l'expression de Srf augmente dans des conditions d'hypertrophie induites par une surcharge de travail, et diminue dans des situations d'atrophie associées à une perte d'activité ou le vieillissement [6-8]. Afin d'élucider le rôle de Srf dans I'hypertrophie musculaire, des souris adultes, chez lesquelles on peut induire l'invalidation du gène Srf uniquement dans les fibres musculaires, ont été soumises à un protocole d'hypertrophie compensatoire (HC) par surcharge expérimentale du muscle plantaris ${ }^{1}(\mathrm{PL})$. Après l'excision du gène Srf, le plantaris

\footnotetext{
${ }^{1}$ Un muscle du compartiment postérieur et superficiel de
} la patte. 


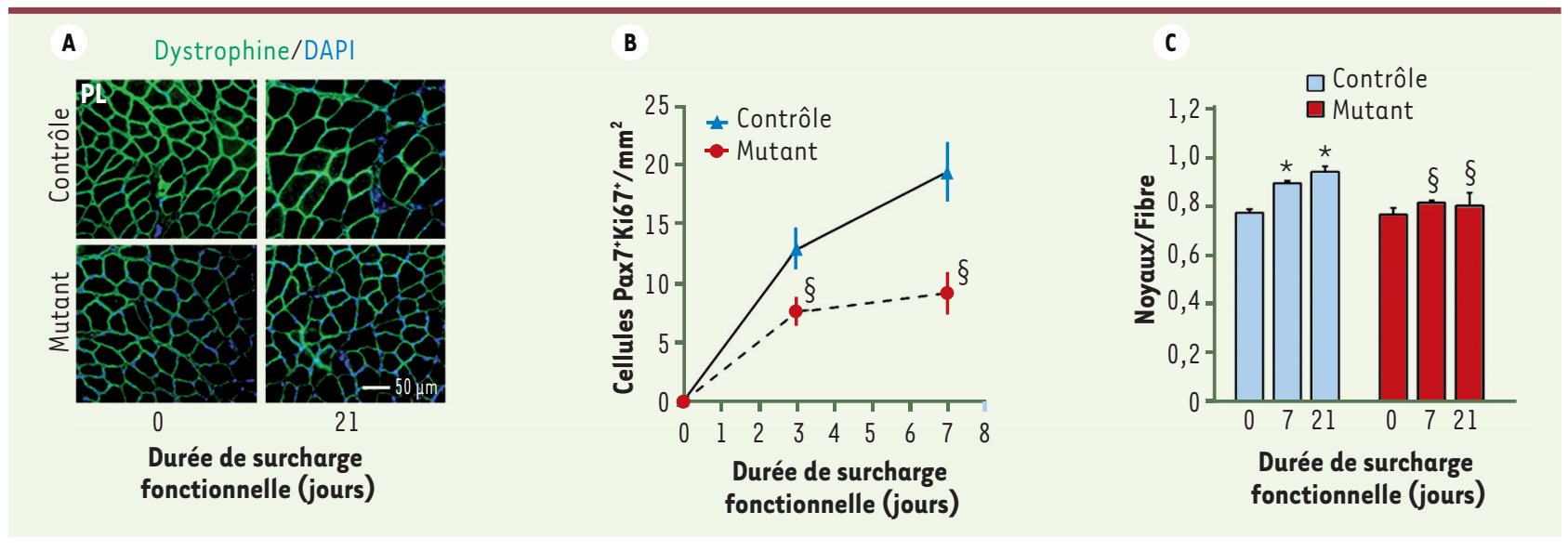

Figure 1. Absence d'hypertrophie des muscles dépourvus de Srf. Les muscles plantaris (PL) exprimant (contrôle) ou non Srf (mutant) ont été soumis à une surcharge fonctionnelle (HC) pendant 3, 7 ou 21 jours. A. Immunomarquage de sections transversales de muscles pour la dystrophine (les noyaux sont révélés par le DAPI) avant (0) et 21 jours après la surcharge. B. Quantification du nombre de cellules Pax $7^{+} \mathrm{Ki} 67^{+}$par $\mathrm{mm}{ }^{2}(\operatorname{Pax} 7 \mathrm{est}$ un marqueur de cellules satellites et Ki67 un marqueur de cellules en prolifération) dans les muscle PL contrôles et mutants 3 à 7 jours après $H C$. Moyenne $\pm S \varepsilon M$. $\S p<0,05$ versus contrôles. C. Quantification du nombre de noyaux DAPI localisés à l'intérieur du sarcolemme (marquage dystrophine) avant ( 0 ) et 7 à 21 jours après HC. Moyenne $\pm S E M .{ }^{*} p<0,05$ versus $(0) ; \S p<0,05$ versus contrôles.

des souris mutantes adultes soumises à une surcharge n'est plus capable de développer une réponse hypertrophique, ce qui montre que Srf est nécessaire à cette réponse adaptative (Figure $1 A$ ).

Une observation plus inattendue était qu'en l'absence de Srf dans les fibres musculaires, les fonctions des cellules satellites étaient perturbées: diminution de leur prolifération (évaluée par le nombre de cellules $\operatorname{Pax} 7^{+} / K i 67^{+}$, Figure $1 B$ ) et de leur fusion aux fibres en croissance (évaluée par le nombre de noyaux par fibre, Figure 1C). Dans le modèle mutant utilisé, la recombinase Cre permettant l'excision du gène Srf n'est pas exprimée dans les cellules satellites et seules les fibres musculaires sont dépourvues de Srf. Ces résultats suggèrent un contrôle paracrine, par la fibre musculaire, de la prolifération et de la fusion des cellules satellites. Nous avons pu démontrer que c'était bien le cas dans des cultures primaires de cellules musculaires mimant la situation in vivo.

\section{Srf contrôle l'expression de l'IL-6, I'IL-4 et Cox2}

Afin d'identifier la nature des molécules qui, sous le contrôle de Srf, sont sécrétées par les fibres musculaires dans des conditions d'hypertrophie, nous avons utilisé une approche globale permettant la mise en évidence de gènes dont l'expression est augmentée en réponse à une activation de Srf dans les myotubes. Nous avons focalisé notre attention sur les gènes codant pour les facteurs sécrétés IL-6, IL-4 ou pour des protéines essentielles à la synthèse de molécules sécrétées comme Cox2 (une enzyme clé de la synthèse des prostaglandines). Nous avons mis en évidence dans les fibres musculaires un réseau de gènes au sein duquel Srf module l'expression d'IL-6 d'une part, et d'IL-4 d'autre part, via le contrôle direct de l'expression de Cox2. Les expressions dans la fibre musculaire d'IL-6, d'IL-4 et de Cox2, contrôlent ainsi respectivement la prolifération et la fusion des cellules satellites.

Afin de montrer que ce mécanisme de régulation est responsable in vivo de l'absence de réponse hypertrophique des muscles dépourvus de Srf, nous avons surexprimé in vivo l'IL-6, l'IL-4 ou Cox2 via l'injection intramusculaire de vecteurs viraux (AAV, adeno-associated virus) recombinants, dans des muscles plantaris de souris mutantes qui étaient ensuite soumises à une surcharge. L'expression d'IL-6 dans les muscles dépourvus de Srf a permis de normaliser la prolifération des cellules satellites, mais n'a pas eu d'effet sur la capacité de ces dernières à fusionner avec les fibres, ni sur la croissance globale du muscle. A contrario, la surexpression d'IL-4 ou de Cox2 n'affecte pas la prolifération de cellules satellites, mais leur permet de fusionner aux fibres et assure la croissance globale du muscle. Ces résultats montrent que le défaut de recrutement des cellules satellites observé dans les muscles dépourvus de Srf est l'évènement limitant pour la croissance et qu'il est relayé par l'IL-4 et Cox2. En effet, la seule restauration de la fusion des cellules satellites aux fibres en croissance est suffisante pour rétablir la croissance musculaire.

Ainsi, Srf semble être un régulateur clé de l'hypertrophie physiologique du muscle squelettique : en réponse à un signal mécanique, Srf stimule, par un mécanisme paracrine, la prolifération des cellules satellites et leur fusion aux fibres musculaires en croissance (Figure 2) [9]. Il est intéressant de remarquer que lorsque nous induisons I'hypertrophie musculaire par l'activation de la voie Akt, donc en l'absence d'une augmentation du signal mécanique, il n'y a pas de recrutement de cellules satellites et Srf n'intervient 


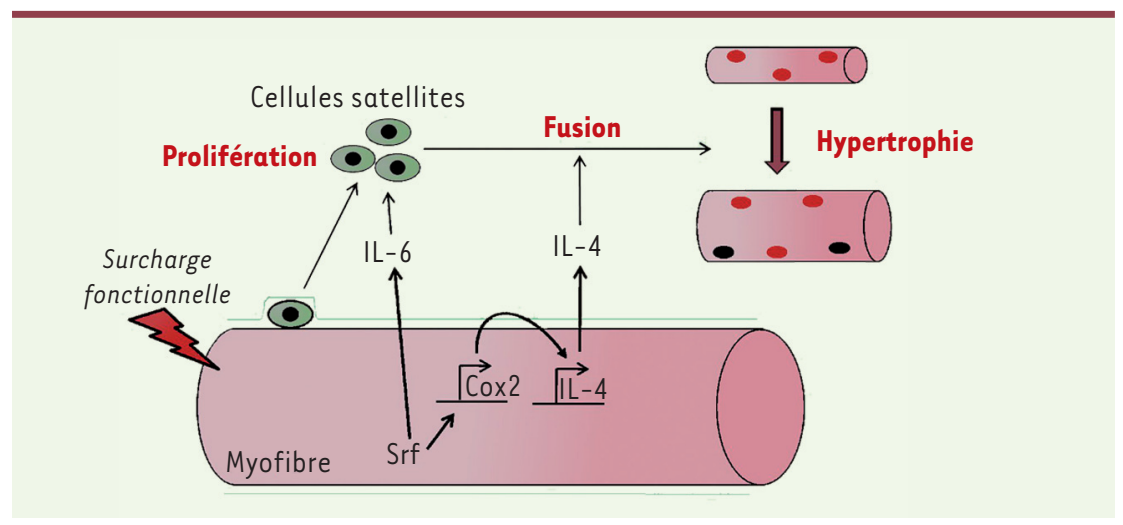

Figure 2. Modèle schématique. En réponse à une surcharge de travail du muscle, Srf dans les myofibres module l'expression de l'IL-6, de Cox2 et de l'IL-4, ce qui permet un contrôle paracrine de la prolifération des cellules satellites pour l'IL-6 et de leur fusion aux myofibres en croissance pour les deux autres facteurs.

pas fonctionnellement. II reste maintenant à élucider la nature des signaux mécaniques interprétés par Srf dans la fibre musculaire et ensuite «traduits » en signaux paracrines pour les cellules souches.

\section{Des pistes pour atténuer la fonte} musculaire au cours du vieillissement? Au cours du vieillissement, on observe une fonte musculaire importante (sarcopénie) accompagnée d'une atténuation de la réponse hypertrophique qu'induit une surcharge fonctionnelle $[10,11]$. Nos études précédentes ont montré, chez la souris comme chez l'homme, une diminution naturelle importante des quantités de Srf au cours du vieillissement [8]. Ceci suggère un lien supplémentaire entre l'activité de Srf et les capacités de croissance du muscle au cours du vieillissement. Ainsi l'identification de Srf comme acteur clé de l'hypertrophie musculaire permet d'envisager de nouvelles voies de recherche pour tenter de contrecarrer l'atrophie musculaire et la sarcopénie. $\diamond$ Srf: a key factor controlling skeletal muscle hypertrophy by enhancing the recruitment of muscle stem cells

\section{LIENS D'INTÉRÊT}

Les auteurs déclarent n'avoir aucun lien d'intérêt concernant les données publiées dans cet article.

\section{RÉFÉRENCES}

1. Glass DJ. Signaling pathways perturbing muscle mass. Curr Opin Clin Nutr Metab Care 2010 ; 13 : 225-9.

2. Kuang S, Gillespie MA, Rudnicki MA. Niche regulation of muscle satellite cell self-renewal and differentiation. Cell Stem Cell $2008 ; 2$ : 22-31.

3. Serrano AL, Baeza-Raja B, Perdiguero $\varepsilon$, et al. Interleukin-6 is an essential regulator of satellite cell-mediated skeletal muscle hypertrophy. Cell Metab $2008 ; 7: 33-44$

4. Horsley V, Jansen KM, Mills ST, Pavlath GK. IL-4 acts as a myoblast recruitment factor during mammalian muscle growth. Cell $2003 ; 113: 483-94$.

5. Charvet C, Houbron C, Parlakian A, et al. New role for serum response factor in postnatal skeletal muscle growth and regeneration via the interleukin 4 and insulin-like growth factor 1 pathways. Mol Cell Biol $2006 ; 26: 6664-74$.

6. Fluck M, Carson JA, Schwartz RJ, Booth FW. SRF protein is upregulated during stretch-induced hypertrophy of rooster ALD muscle. J Appl Physiol $1999 ; 86$ : 1793-9.

7. Lamon S, Wallace MA, Leger B, Russell AP. Regulation of STARS and its downstream targets suggest a novel pathway involved in human skeletal muscle hypertrophy and atrophy.J Physiol 2009 ; 587 : 1795-803.

8. Lahoute C, Sotiropoulos A, Favier M, et al. Premature aging in skeletal muscle lacking serum response factor. PLoS One 2008 ; 3 : e3910.

9. Guerci A, Lahoute C, Hébrard S, et al. Srf-dependent paracrine signals produced by myofibers control satellite cell-mediated skeletal muscle hypertrophy. Cell Metab 2012; 15 : 25-37.

10. Alway SE, Degens H, Krishnamurthy G, Smith CA. Potential role for Id myogenic repressors in apoptosis and attenuation of hypertrophy in muscles of aged rats. Am J Physiol Cell Physiol 2002 ; 283 : C66-76.

11. Carson JA, Yamaguchi M, Alway SE. Hypertrophy and proliferation of skeletal muscle fibers from aged quail. J Appl Physiol 1995 ; 78 : 293-9.
NOUVELLE

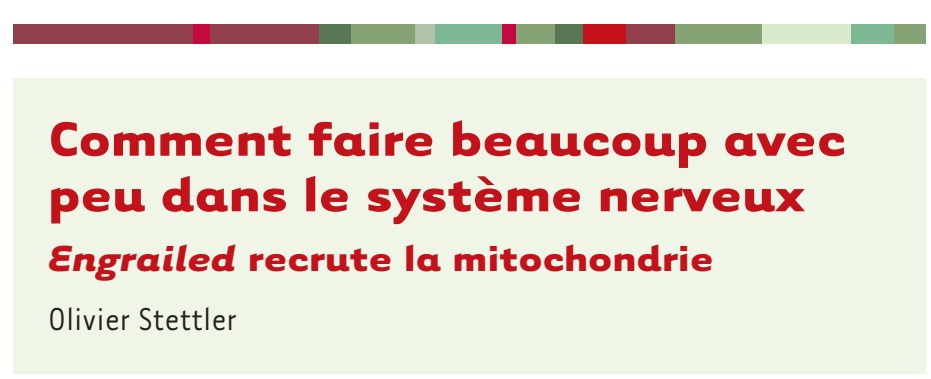

> Lors du développement cérébral chez l'homme, pas moins de 100 milliards de neurones doivent établir chacun jusqu'à 100000 connexions synaptiques avec d'autres cellules nerveuses. Le bon fonctionnement du cerveau dépend de la précision avec laquelle les axones, qui vont établir ces connexions, sont préalablement guidés vers leurs territoires cibles. Le guidage axonal est contrôlé par des molécules extracellulaires agissant en général par l'intermédiaire de récepteurs associés à la membrane plasmique du cône de croissance, une structure sensorimotrice située à l'extrémité des axones en élongation
UMR CNRS 8192, laboratoire de dynamique membranaire et maladies neurologiques, université Paris Descartes, 45, rue des Saints-Pères, 75005 Paris, France. olivier.stettler@parisdescartes.fr

[1]. Ces molécules de guidage exercent des effets attractifs ou répulsifs sur l'axone en modifiant la dynamique du cytosquelette du cône de croissance. Dès les années 1960, Sperry introduit le concept d'une connectivité cérébrale s'établissant sous l'action combinée de molécules de guidage spécifiques. 